\title{
Sensor-Based Data Storage for Search and Rescue
}

\author{
Sanem Sariel Talay ${ }^{1}$, Esin Ergen ${ }^{2}$, Gurhan Avdan ${ }^{1}$ and Cagri Eroglu ${ }^{1}$ \\ ${ }^{1}$ Computer Engineering Department, Istanbul Technical University \\ Electrical and Electronics Faculty, Maslak, Istanbul, Turkey, 34469,\{sariel,avdan,erogluc\}@itu.edu.tr \\ ${ }^{2}$ Civil Engineering Department, Istanbul Technical University \\ Insaat Fakultesi, Maslak, Istanbul,Turkey,34469, esin.ergen@itu.edu.tr
}

\begin{abstract}
This research study proposes to utilize sensors for making necessary local information readily available and easily accessible to search and rescue team members following an earthquake. The Search and Rescue Data Access Point (SR$D A P)$ system is designed for storage and retrieval of useful local information on/from sensor nodes deployed on building frontages. In the paper, the types of information items that are needed during search and rescue operations are determined, and the requirements identified for the proposed system are discussed. Then, the representation of these items and the design of SR-DAP are presented. The discussions on the current information needs are based on the search and rescue guidelines and on the initial interviews that were conducted with earthquake responders in Turkey such as Turkish Civil Defense Association and AKUT (Turkey Search and Rescue Association). The prototype system has been designed and implemented by using real sensors.
\end{abstract}

\section{INTRODUCTION}

In case of an earthquake, urban search and rescue operations are performed to locate and rescue victims who are trapped in collapsed structures and to provide immediate medical treatment. A critical factor that affects the efficiency and the effectiveness of search and rescue efforts is the availability of information related to buildings and their residents. Following an earthquake, it is difficult to access this local information from centralized databases because information infrastructure is usually damaged, unreliable, or overloaded [1], [2]. Therefore, search and rescue teams collect the related local information from either local residents or the disaster environment intuitively. For example, according to Federal Emergency Management Agency's (FEMA) search and rescue guidelines [3], one of the search and rescue team members needs to walk around the damaged building to determine approximate dimensions of the building while another member talks to local people to figure out how many victims might be in the building. The collected information is unreliable and incomplete as it is intuitively collected and the people who provide the information are likely to be still under trauma due to recent earthquake experience. Furthermore, gathering local information by using these traditional methods is time-consuming. Since probability of rescuing people under a collapse decreases $50 \%$ or more after a 24 hour period [3], a more effective and reliable approach for local data collection is needed to decrease fatalities.

The objective of the ongoing research study explained in this paper is to develop a local data storage system that provides the required information for effective search and rescue operations. The research study proposes to utilize sensors for making necessary local information readily available and easily accessible to search and rescue team members following an earthquake. This paper discusses the types of information items that are collected during search and rescue operations and presents the architecture of the Search and Rescue Data Access Point (SR-DAP) system for storage and retrieval of the necessary local information on/from sensor nodes deployed on building frontages. The discussions on the current information needs are based on the search and rescue guidelines and on the interviews that were conducted with experts in earthquake response in Turkey such as Turkish Civil Defense Association and AKUT (Turkey Search and Rescue Association).

\section{MotivATION AND BACKGROUND}

Five phases are defined for search and rescue operations at earthquake incidents by the earthquake response guidelines and the search and rescue experts that were interviewed [3]:

- Assessment and command of the area: In this phase, rescue personnel conduct an initial survey to collect information about the victims and the structures, and perform reconnaissance of the area by walking around structures. The collected information is assessed and, building triage is conducted to evaluate the integrity of the buildings and determine search priorities in the area.

- Removal of surface victims: After hazard assessment of a structure, rescuers remove surface victims as safely and quickly as possible.

- Search for viable victims in voids and accessible spaces: Rescuers search and explore all voids and accessible spaces for viable victims.

- Selected debris removal: The team removes the selected debris using special tools or techniques.

- The entire debris field is removed.

The first phase starts with information gathering about the area to conduct building triage which is followed by the search for victims. Building triage is ranking the damaged 
buildings to decide where to start search and rescue and determine how the resources will be allocated. To identify the buildings with highest potential for rescue, it is important to know the function of the buildings in the neighborhood (e.g., school and apartment building). Based on the function of the building and the damage observed, the possible number of victims is determined for each building. If possible, any available information about the number, conditions and locations of the victims is also considered while performing building triage. Existence of hazards in the building might cause the building to have a low priority in rescue operations. Because, hazards (e.g., hazardous materials) might endanger the lives of rescuers or delay the search and rescue operations until the required personnel and equipments are obtained.

Once a building is selected for search and rescue operations, the search and rescue teams need to know about the building layout and design (e,g., location of stairwells and elevator shafts) to identify the possible locations and voids where people might be trapped. To collect this information, a member of a search and rescue team walks around the damaged building and makes a rough sketch of the building, highlighting outstanding features. In the meantime, another team member talks to people in the neighborhood to gather information about the building, its contents and its residents. If possible, information about the utility shut-off locations is also collected to turn off the utilities before entering the building for preventing any hazards.

It is time-consuming to collect information through observations at site and by talking to people in the neighborhood who are familiar with the buildings and witnessed the disaster. Also, information provided by local people is not reliable. These issues highlight a need for local information access points. The information made available at those access points enables the search and rescue teams to quickly retrieve some of the necessary information which can be used to complete search and rescue operations more effectively in a shorter amount of time. Moreover, the search and rescue teams that were interviewed stated that existence of local data storage units might provide opportunities for storing other information items that are not currently available, such as building pictures or model, name of each person and their health information.

Existing disaster management systems are mostly developed for identification, prediction and recovery phases and do not support real-time response activities [4]. For example, HAZUS (Hazards United States) [5] and HAZTURK (Hazards Turkey) [6] are risk assessment tools, CATS (consequence assessment tool set) was developed for predicting hazards and disaster analysis, and a commercially available program is used in recovery phase for post disaster data management [4].

In the recent studies, frameworks for disaster management systems that cover the response phase are developed [4], [7],
[8]. To improve collaboration among responders, some researchers focused only on the response phase and proposed to use frameworks that do not rely on an infrastructure and use ad-hoc wireless communication [7], [8]. These frameworks incorporate various components such as GIS, web collaboration tools, robots, sensors, RFID tags and building black box systems. Most of the research studies related to those frameworks focus on establishing an effective ad-hoc communication among mobile devices used by the responders.

As a part of the framework proposed by Chen et al. [8], Tsai et al. [9] propose a conceptual building black box system that integrates with other advanced building sensing and control systems. This black box system provides necessary information about the building and its residents for use during search and rescue.

The ongoing research explained in this paper builds on and extends the proposed black box system [9]. Instead of having a complex building black box network, integrated with advanced building sensing and control systems, a sensorbased local data storage system is designed. The reason for such a design is that advanced building sensing and control systems do not exist in current buildings and black box systems cannot be deployed without those systems. A sensorbased local data storage system can be easily deployed at existing buildings without the need for integrating it to other advanced systems.

The novelty of the proposed system is to use sensor networks as local databases that can be accessed by search and rescue teams besides their sensing abilities. The advantage of using a sensor network for storing local information is larger memories (i.e., $1 \mathrm{MB}$ ) and the convenience of accessing and updating the data at any location in the network due to data routing capabilities of the nodes. In this paper, the prototype design of the overall system is presented and a further analysis of the wireless network design of the system and security precautions is left as a future work.

\section{SYSTEM REQUIREMENTS}

In the proposed system, the information items that are needed by a team during search and rescue operations are stored locally on sensors before an earthquake occurs, and these information items are made readily available for use on demand. Search and rescue teams access this information to make effective decisions and to perform search and rescue operations in an efficient way. Once the search and rescue operations are completed in one of the buildings, the search and rescue assessment information is stored into the local data storage unit to document the performed activities for use by other teams.

Sensors are attached to buildings and information that is needed for search and rescue operations is stored into them 
beforehand. The search and rescue teams carry a handheld computer (e.g., PDA) integrated with a sink sensor node to retrieve data from sensor nodes or to store data in them.

The proposed system is designed to initially provide information at neighborhood level and building level. The neighborhood level information is stored at a local public building, for example at a building where registration records of the buildings in the neighborhood and its residents are kept. The neighbor level information gives an overview of the area and helps the search and rescue teams make effective decisions about which buildings have priorities and the highest potential survivors.

Once the building triage is performed and a building for search and rescue is selected, rescuers need to get information related to that building. The building level information is stored locally at each building and provides detailed information about the structure, its contents and its residents. The retrieved information from one of the sensor nodes attached on the building assists the search and rescue teams to perform an effective search and rescue operation in the building.

Having completed the search and rescue operations, search and rescue teams enter assessment information in the accessible sensor nodes. This information is later to be accessed by other search and rescue teams who might check the building for potential survivors or recovery teams who perform the debris removal.

\section{DAta Representation FOR Limited Memory SPACE}

The neighborhood level information includes the transportation plan and the layout plan of the neighborhood that shows utility lines, the locations of shut-off valves and containers that includes supplies for search and rescue operations, the hazardous materials and their locations, building usage types and the total number of people living in each building.

The building level information mainly consists of the following items: the building identification information, the coordinates of the building, the usage type of the building, the number of floors, the type of the structural system (e.g., concrete), the construction year of the building, the number of people staying in the apartments within specific time periods (e.g., during daytime), the hazardous materials, the layout/utility shut-off plans of the building, the pictures of the building from four sides and the personal information of residents in each apartment (e.g., name, photo and health). The building layout plan is used to identify locations where a victim might survive (e.g., basements, rooms next to stairwells) or might get stuck while escaping from the building (e.g., hallways, stairways) or places where the chance of survival is high (e.g., the voids created by heavy machinery or furniture, the locations near chimneys and fireplaces). The building layout plan is also used by search and rescue teams to navigate in the building. Utility shut-off locations are needed to turn off the utilities before entering into the building. When identifying the building level information, the authors limited the research study to residential buildings.

The search and rescue assessment information presents the current search status and search findings, such as hazards, the total number of victims inside the structure, their names and conditions (e.g., alive or dead), the total number of victims rescued, their names, conditions and locations where they were taken, time of the latest assessment, the name of the search team, and the method used for the search.

Since all these information items are proposed to be stored in sensor nodes which have limited memories, low memory requirements should be met. To minimize the storage space needed for all information items, data are encoded into byte streams which are then translated into hexadecimal code for serial transmission. Encoding procedure converts the given user input into encoded form by using local database entries for specific contents, ensuring a means of information storage into the limited external memory of the sensor node. All images taken from the user is converted into jpeg format in which compression is directly applied without data loss. Image files in this form can be stored in the sensor node. Other compression methods are not directly applicable for efficient serial transmission of the data stream.

To illustrate the representation of information, an example for the classification of building types is given in Table 1. In this table, the general usage type of the buildings, one of the information items to be stored in the sensors, are classified and encoded. A sub-classification is also performed for each building type. For example, the classification of educational buildings and the related encodings are given in Table 2 .

TABLE 1: CLASSIFICATION OF BUILDING TYPES
\begin{tabular}{|l|c|}
\hline Usage of the Building & Encoding \\
\hline Residence & 0 \\
\hline Health Structure & 1 \\
\hline Educational Building & 2 \\
\hline Office & 3 \\
\hline Security Organization & 4 \\
\hline Industrial Plant & 5 \\
\hline Mansion & 6 \\
\hline Social Activity Place & 7 \\
\hline Religious Building & 8 \\
\hline
\end{tabular}

TABLE 2: INTERNAL CLASSIFICATION OF EDUCATIONAL BUILDINGS
\begin{tabular}{|l|l|c|}
\hline Usage & Type & Encoding \\
\hline \multirow{4}{*}{ Educational Building } & Kindergarten & 1 \\
\cline { 2 - 3 } & Primary School & 2 \\
\cline { 2 - 3 } & High School & 3 \\
\cline { 2 - 3 } & University & 4 \\
\cline { 2 - 3 } & Schoolroom & 5 \\
\hline
\end{tabular}

Table 3 reports the total amount of memory space needed for the overall building level information. With a proper 
encoding, all information items including the pictures of the building and the residents can be stored in $661 \mathrm{kB}$ memory space which is a reasonable amount of memory space for a sensor node. The same procedure is also applied for both calculating the neighborhood level information and the search and rescue assessment information.

TABLE 3: MEMORY SPACE NEEDED FOR BUILDING LEVEL INFORMATION

\begin{tabular}{|l|c|}
\hline Information item & $\begin{array}{c}\text { Amount of } \\
\text { storage }\end{array}$ \\
\hline Building identification information & $200 \mathrm{bits}$ \\
\hline Coordinates of the building & $40 \mathrm{bits}$ \\
\hline Usage type of the building & $7 \mathrm{bits}$ \\
\hline Number of floors & $6 \mathrm{bits}$ \\
\hline Structural system & 2 bits \\
\hline Construction date (Year) & 12 bits \\
\hline $\begin{array}{l}\text { Number of people within specific time periods (for 100 } \\
\text { individuals) }\end{array}$ & 35 bits \\
\hline Hazardous materials (5) & 135 bits \\
\hline Building layout/Utility shut-off plans (4 types) & $300 \mathrm{kB}$ \\
\hline Pictures of the building (4 sides) & $40 \mathrm{kB}$ \\
\hline $\begin{array}{l}\text { Personal information (Identification and medical } \\
\text { information for 100 individuals) }\end{array}$ & $21 \mathrm{kB}$ \\
\hline Pictures of people living in the building (for 100 person) & $300 \mathrm{kB}$ \\
\hline Total memory space needed & $\mathbf{6 6 1 ~ k B}$ \\
\hline
\end{tabular}

\section{Overall System Design}

The Search and Rescue Data Access Point (SR-DAP) system is designed for storage and retrieval of local information on/from sensor nodes deployed on exterior walls of buildings to assist search and rescue operations. Figure 1 illustrates the components of the SR-DAP system. Based on the requirements, three basic phases are envisioned in the system. A local database for encoding purposes is assumed to exist in the computing hardware (i.e., PDAs, personal computers or laptops) of either operators or rescuers.

In the first phase of the system setup, local information for a building is stored in the sensors attached on the building (Figure 1(a)). The building level information is taken by the interface program. Then, the given information is converted into encoded raw hexadecimal data. The translated hexadecimal code is transmitted to the sensors through serial link. On the sensor side, the incoming hexadecimal code is transferred into the flash memory of the sensor node to be available whenever the node is alive. The user input is also transmitted to a database server for auxiliary services (e.g., for use in centralized disaster management systems).
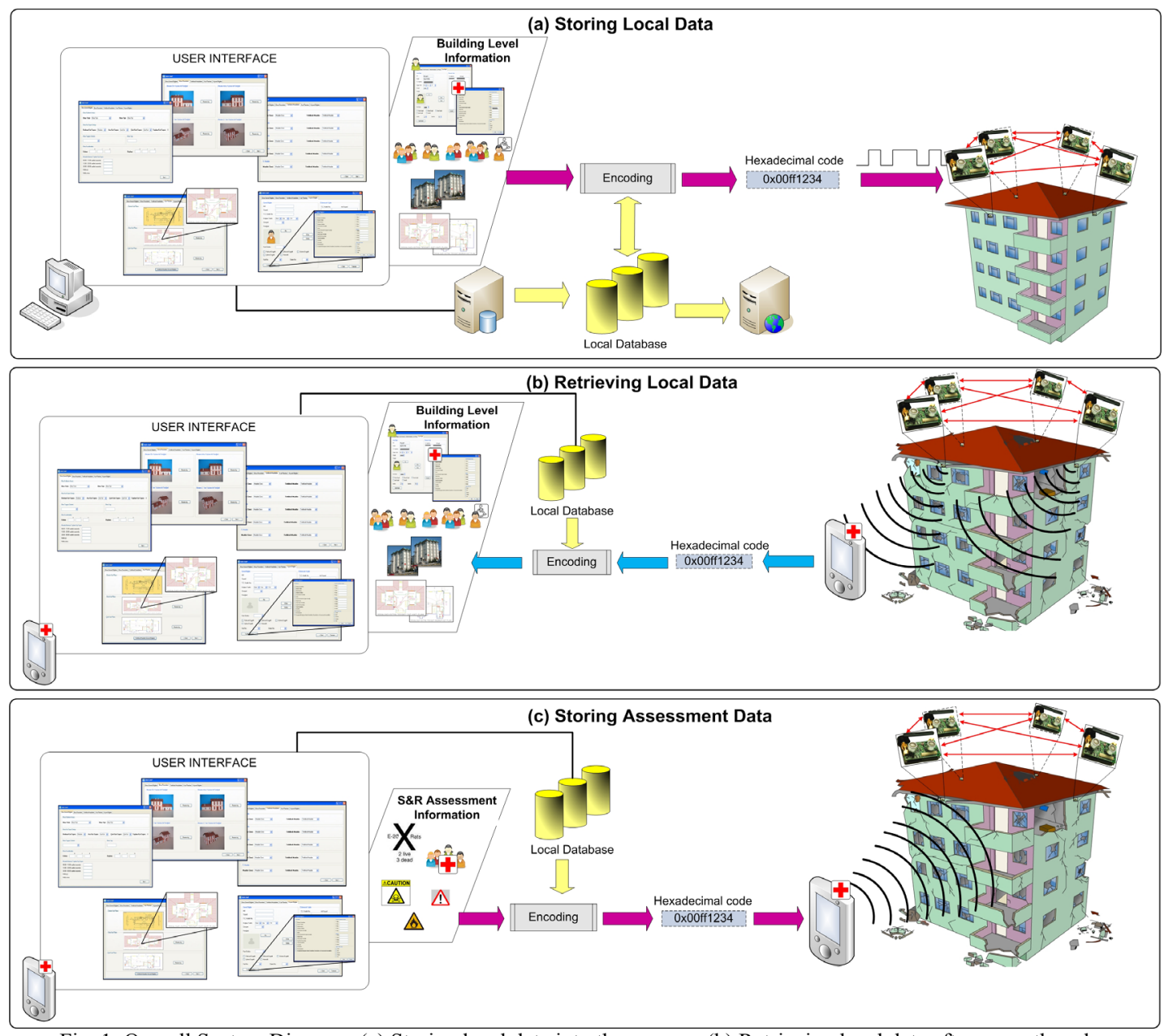

Fig. 1: Overall System Diagram: (a) Storing local data into the sensors, (b) Retrieving local data after an earthquake,

(c) Storing assessment data into the sensors 
In the second phase, after an earthquake, search and rescue members can retrieve the local data associated with a selected building (Figure 1(b)). This selection is usually performed by using neighborhood level information stored in a public building. Some buildings have higher priorities for rescue operations depending on the building type or the number of people in the building when the disaster occurs. Once the search and rescue team members identify the building to be searched, they can retrieve the relevant information from the corresponding sensor attached to the building to effectively search the area by using PDA/laptop-based sensor readers. The data stored in one of the sensors in range are transmitted through the radio link and the relevant information is shown to the rescuer in the user interface. Many sensors of different buildings may be in the range of the rescuer. In this case, the sensor that stores data for the corresponding building can be determined by going through building pictures or coordinates which are stored in the sensors as building level information. Even when the building is heavily damaged, the painting color may help matching the corresponding sensor and the related information with the building to be searched.

In the third phase, the search and rescue assessment information is stored into the nodes by the search and rescue team (Figure 1(c)). Input is taken from the rescuer and the data are converted into hexadecimal code as in the first phase. However, in this case, data transmission to sensor nodes is performed through RF links. The sensors in range and accessible are notified with the assessment information.

\section{IMPLEMENTATION DETAILS}

SR-DAP has been designed and implemented by using Sensenode v.1.3. sensors (Figure 2) in a laboratory environment. Nodes are compatible with IEEE 802.15.4 wireless standard and the TinyOS operating system. The low power key characteristic of the sensors is provided by the MSP430 microcontroller having 10kB RAM and 48kB Flash memory. Sensenode uses the ST M25P80 40MHz serial code flash for external data and code storage. The external flash memory holds $1024 \mathrm{kB}$ of data and is decomposed into 16 segments, each $64 \mathrm{kB}$ in size. Sensenodes have antennas with $80-100 \mathrm{~m}$ range. Onboard chipcon CC240 radio technology provides a $250 \mathrm{kbps}$ data rate at $2.4 \mathrm{GHz}$ frequency [9]. Embedded programs for sensors are written in nesC programming language running on TinyOS operating system.

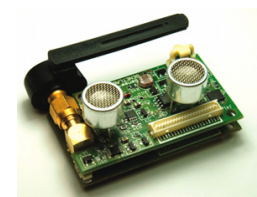

Fig. 2: Genetlab Sensenode v.1.3.

Data storage is the most important phase during the implementation of SR-DAP. During the serial transmission and storage, the data coming from the serial port is written into the flash (external) memory of the node as 256 byte packages. A 256 byte data buffer, defined in the program memory of the sensor, is used as a means of a temporary storage space for the incoming data from the serial port. Piecewise data storage is needed due to single-line serial transmission limitations. To implement piecewise storage, writing into the external memory and reading from the serial port is performed separately. Serial transmission is interrupted by receiving a special stop character, indicating a 256 byte data package has arrived from the user interface. This package is written into the flash memory. Then, serial transmission continues periodically for the remaining data packages. The flow of data through the serial link between a computer and a sensor is illustrated in Figure 3 for both directions. Data transmission for wireless communication between sensors is also performed in the same manner.

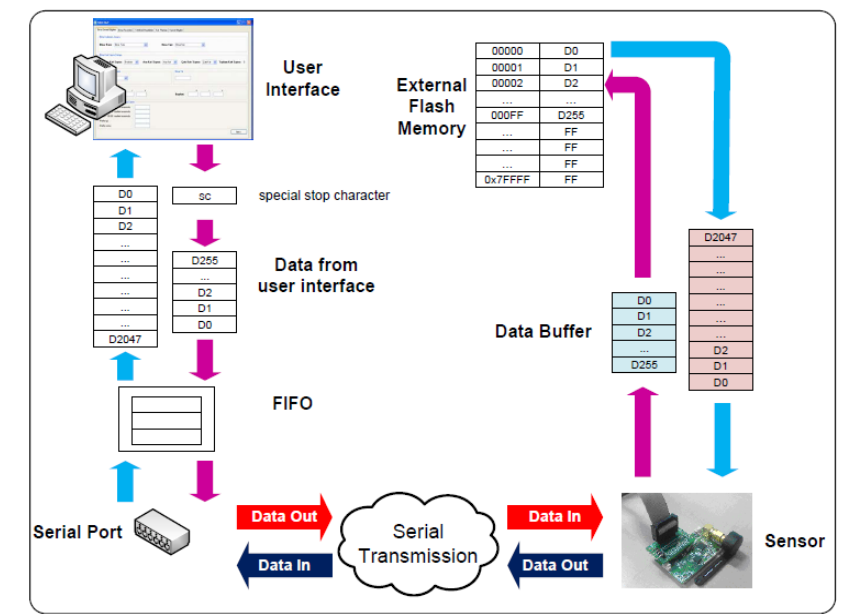

Fig. 3: Overview of data transmission through the serial link to store/retrieve data on/from the Sensenode v.1.3.

\section{DisCUSSION}

SR-DAP has been designed and implemented to meet the design objectives and the requirements identified for search and rescue operations following an earthquake. The required information related to local buildings and residents are proposed to be stored in sensors attached to outer surfaces of buildings. These sensors would also be used for storing search and rescue assessment information. The system employs three phases for (1) storage of the building and neighborhood level information, (2) retrieval of the stored information and (3) storage of the search and rescue assessment information in sensors. In the worst case scenario, although the nodes are encapsulated and protected against impacts and pressure, some of them may be damaged during the earthquake and the information stored in them would be lost. To prevent data loss, redundant data storage is needed. Thus, building level information would be replicated in sensors attached on the same building. Another solution for data redundancy is to have one node in each building and to store information that belongs to multiple buildings in that node. For example, a node might not only contain information that belongs to the building which the node is attached to, but also include information that belongs to buildings in close 
proximity. In either case, the sensor network in the district would guide rescuers for the selection of the building to be searched in the neighborhood level.

Storage of a large amount of information is made possible by a proper encoding and compression scheme in the system. However, when the amount of information to be stored exceeds the amount of the memory space of sensors, data could be distributed over sensors in a network for each building. In this case, retrieval is performed by collecting all the information distributed over the network. Energy consumption schemes may be applied by using special storage sensors [11].

Tagging each resident with a sensor and/or allowing dynamic content for the building level information is possible. For example, instead of storing static information for the number of people at specific time periods, this information could be easily retrieved by an efficient tracking mechanism. A similar approach is used for tracking lost hikers [12]. However, these techniques are not usually preferred publicly due to privacy concerns. SR-DAP system, thus, has been designed accordingly to address this issue.

Sensors are used to utilize local data storage in SR-DAP. Depending on either $\mathrm{CO}_{2}$ or heat sensing performance of the sensors, the localization of the victims trapped in buildings is also planned to be dynamically done by using sensing capabilities of the sensors.

\section{Conclusion}

This paper outlines the design and the implementation of SRDAP for storage and retrieval of local information on/from sensor nodes deployed on exterior walls of buildings to improve search and rescue operations. The system assists the rescue teams to easily and effectively access the necessary local information on demand after an earthquake. This would help reducing the time spent for search and rescue activities and consequently increasing the number of people who are rescued after an earthquake.

The research study described in this paper is an ongoing work and the tests are currently being conducted in a laboratory environment. The near future work includes the field experiments of the designed system in a disaster like environment, and then the actual deployment of the system at existing buildings in urban areas. For the actual deployment, building collapse mechanisms need to be investigated and the locations that are least damaged in buildings should be identified as appropriate locations for attaching the nodes.

\section{ACKNOWLEDGEMENT}

Authors would like to thank Gursans Guven and Senem Seyis for their tremendous efforts in conducting interviews and helping outline the data model of the system. This research is funded by a grant from the Scientific and Technological Research Council of Turkey (TUBITAK), Grant No. 107M144. TUBITAK's support is gratefully acknowledged. Any opinions, findings, conclusions or recommendations presented in this paper are those of the authors and do not necessarily reflect the views of the TUBITAK.

\section{REFERENCES}

[1] The World Bank, "Turkey - Marmara earthquake assessment", Turkey Country Office, http://siteresources.worldbank.org, September 14, 1999.

[2] Ozerdem, A. and Jacoby T., "Disaster Management and Civil Society: Earthquake Relief in Japan, Turkey and India", I B Tauris \& Co Ltd, 2005.

[3] FEMA 2003: U.S. Government, Progressive Management, "21st Century Complete Guide to FEMA”, 2003.

[4] Pradhan, A, Laefer, D. F., and Rasdorf, W. J., "Infrastructure Management Information System Framework Requirements for Disasters", Journal Of Computing In Civil Engineering, vol. 21 (2), pp. 90101, 2007.

[5] FEMA 2007: "HAZUS - FEMA's Software Program for Estimating Potential Losses from Disasters", http://www.fema.gov, 2007.

[6] Elnashai, A.S., Hampton, S., Karaman, H., Sung Lee, J., Mclaren, T., Myers, J., Navarro, C., Sahin, M., Spencer, B., Tolbert, N., "Overview and Applications of MAEviz-Istanbul (HAZTURK)", International Symposium on Loss Estimation for Turkey, 2007.

[7] Asama, H. Hada, Y. Kawabata, K. Noda, I. Takizawa, O. Meguro, J. Ishikawa, K. Hashizume, T. Ohga, T. Hatayama, M. Matsuno, F. Todokoro, S., "Rescue Infrastructure for Global Information Collection", SICE-ICASE, International Joint Conference, 2006.

[8] Chen, A.Y., Tsai, M. H., Lantz, Timothy S., Plans, A. P., Mathur, S., Kaushik, N., Lakhera, S., and Feniosky, P. M., "A Collaborative Framework for Supporting Civil Engineering Emergency Response with Mobile Ad-Hoc Networks", ASCE International Workshop on Computing in Civil Engineering, 2007.

[9] Tsai, M.-H., Liu, L. Y., Pena-Mora, F., "A Building Black Box for Urban Disaster Response and Relief," ASCE International Workshop on Computing in Civil Engineering, 2007.

[10]Genetlab, Sensenode Technical Specifications Document v1.3b, 2008

[11] Sheng B., Li Q. and Mao W., "Data storage placement in sensor networks", International Symposium on Mobile Ad Hoc Networking \& Computing, 2006

[12]Huang J., Amjad S., Mishra S., "CenWits: A SensorBased Loosely Coupled Search and Rescue System Using Witnesses", The $3^{\text {rd }}$ ACM Conference on Embedded Networked Sensor Systems 2005. 